\title{
Optoelectronic and Microwave Transmission Characteristics of Indium Solder Bumps for Low-Temperature Flip-Chip Applications
}

\author{
Kun-Mo Chu, Student Member, IEEE, Jung-Hwan Choi, Jung-Sub Lee, Han Seo Cho, Seong-Ook Park, \\ Hyo-Hoon Park, Member, IEEE, and Duk Young Jeon
}

\begin{abstract}
This paper describes low-temperature flip-chip bonding for both optical interconnect and microwave applications. Vertical-cavity surface-emitting laser (VCSEL) arrays were flip-chip bonded onto a fused silica substrate to investigate the optoelectronic characteristics. To achieve low-temperature flip-chip bonding, indium solder bumps were used, which had a low melting temperature of $156.7^{\circ} \mathrm{C}$. The current-voltage $(I-V)$ and light-current $(L-I)$ characteristics of the flip-chip bonded VCSEL arrays were improved by Ag coating on the indium bump. The $I-V$ and $L-I$ curves indicate that optical and electrical performances of Ag-coated indium bumps are superior to those of uncoated indium solder bumps. The microwave characteristics of the solder bumps were investigated by using a flip-chip-bonded coplanar waveguide (CPW) structure and by measuring the scattering parameter with an on-wafer probe station for the frequency range up to $40 \mathrm{GHz}$. The indium solder bumps, either with or without the Ag coating, provided good microwave characteristics and retained the original characteristic of the CPW signal lines without degradation of the insertion and return losses by the solder bumps.
\end{abstract}

Index Terms-Coplanar waveguide (CPW), flip-chip bonding, indium bump, optical interconnection, silver, solder, vertical-cavity surface-emitting laser (VCSEL).

\section{INTRODUCTION}

$\mathbf{F}$ LIP-CHIP interconnection is becoming a popular technique for packaging of optoelectronic and microwave devices. The advantages of flip-chip technology with bumps include the reduction of interconnection length, resistance, and inductance in high-frequency and wide-band systems. Especially, flip-chip interconnection provides an excellent

Manuscript received February 1, 2005; revised June 8, 2005. This work was supported in part by the Center for Electronic Packaging Materials (ERC) of MOST/KOSEF under Grant R11-2000-085-10001-0 and in part by the Korea Ministry of Science and Technology (MOST) under the national program for tera-level nanodevices as one of the " 21 st century frontier R\&D projects."

K.-M. Chu, and J.-S. Lee are with the Department of Materials Science and Engineering, Korea Advanced Institute of Science and Technology, Daejeon 305-701, Korea, and also with the Optical Interconnection and Switching Laboratory, Information and Communications University, Daejeon 305-714, Korea.

J.-H. Choi and S.-O. Park are with the Microwave and Antenna Laboratory, Information and Communications University, Daejeon 305-714, Korea.

H. S. Cho is with the Optical Interconnection and Switching Laboratory, Information and Communications University, Daejeon 305-714, Korea. He is also with the Samsung Electro-Mechanics Corporation, Ltd., Yeongi-Gun Chungnam 339-702, Korea.

H.-H. Park is with the Optical Interconnection and Switching Laboratory, Information and Communications University, Daejeon 305-714, Korea.

D. Y. Jeon is with the Department of Materials Science and Engineering, Korea Advanced Institute of Science and Technology, Daejeon 305-701, Korea. Digital Object Identifier 10.1109/TADVP.2006.875417 electrical behavior up to the millimeter-wave range in microwave devices [1]. For flip-chip bonding, the commonly used solders are $\mathrm{Au}-\mathrm{Sn}$ and $\mathrm{Pb}-\mathrm{Sn}$ alloys. These materials, however, are unsuitable for optoelectronic hybrid systems in which high-temperature processing can degrade optical devices, such as polymeric waveguides or microlenses. In particular, for acrylate series polymeric waveguides, low-temperature solders that can be processed below $160{ }^{\circ} \mathrm{C}$ are recommended [2]. Alloys containing indium are widely used in applications for which relatively low temperature processing is required. In addition, due to its ductility, indium solder has become increasingly prevalent in the implementation of photonics, fiber-optic devices, and packaging [3]. However, during soldering operations, indium easily forms oxides that have very high melting point. The solid oxide film adheres onto the molten solder and thus prevents the solder solution from contacting with the surface to be joined [4]. Consequently, quality joints cannot be achieved, and the adhesion strength between indium bump and chip pad becomes weak. In our previous work [5], for flip-chip bonding of a vertical-cavity surface-emitting laser (VCSEL) array chip on glass substrate, a thin Ag coating on the indium bumps found to be very effective at both preventing oxidation of the indium bumps and decreasing the melting temperature of the pure indium solder. The result was enhanced adhesion strength between the indium bumps and the VCSEL chip pads.

This paper investigates the effects of a Ag coating on the indium bump with respect to optoelectronic and microwave performances. These performances are analyzed using two kinds of test samples such as an optical interconnection plate and a coplanar waveguide (CPW) package.

\section{EXPERIMENT}

To measure the optoelectronic and microwave characteristics of the solder bumps, two kinds of interconnection samples were prepared. The optoelectronic characteristics were tested for a sample in which the 850-nm VCSEL arrays were flip-chip bonded onto the fused silica substrate by means of solder bumps. The microwave characteristics of the solder bumps were investigated with a flip-chip-bonded CPW structure.

\section{A. Fabrication of Optical Interconnection Samples}

The application of low-temperature flip-chip bonding is targeted to the optical interconnection plate [2] where a thermally weak polymeric waveguide is integrated with optical devices, as 


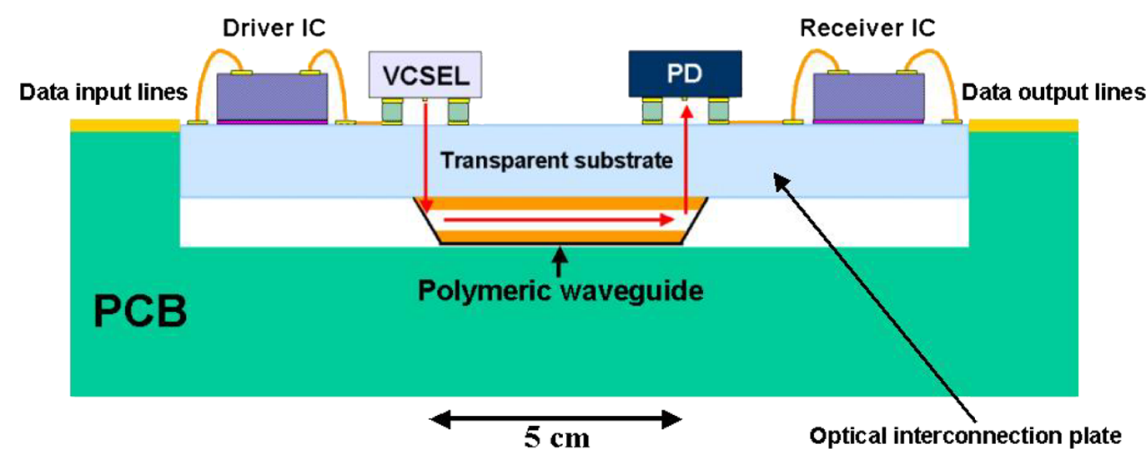

Fig. 1. Architecture of the optical interconnection plate assembled with a conventional PCB. (Color version available online at http://ieeexplore.ieee.org.)

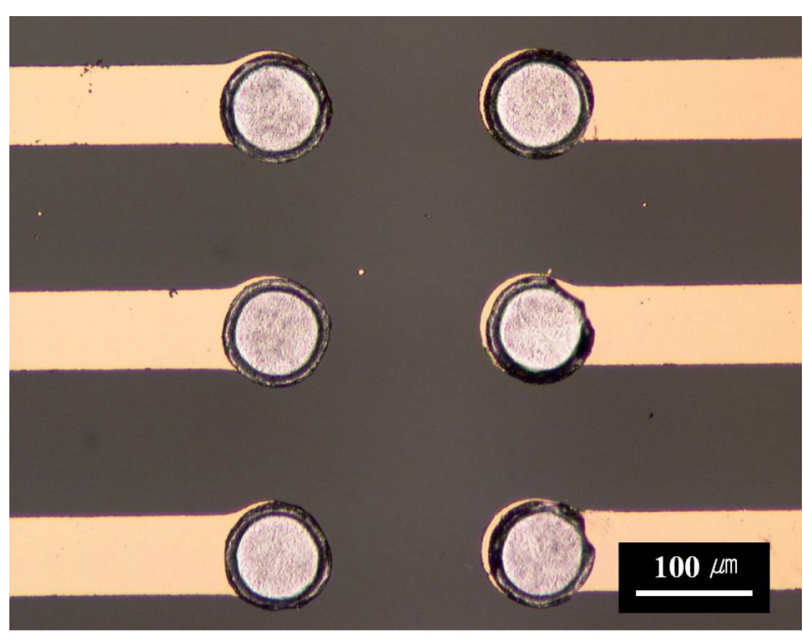

(a)

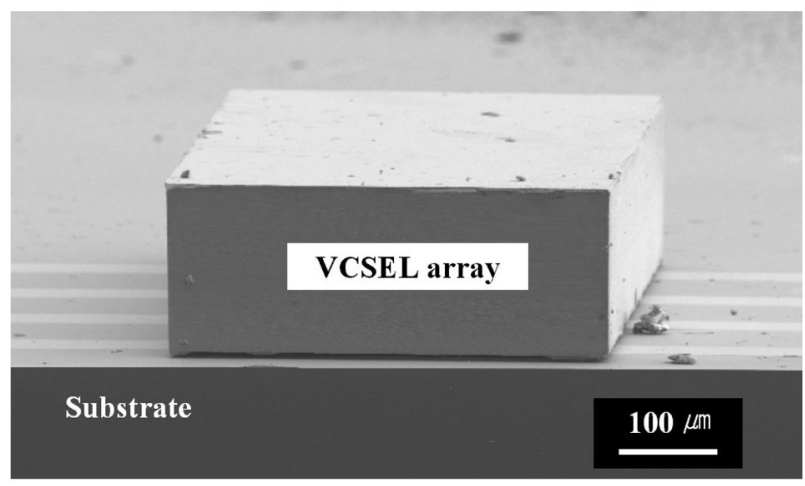

(b)

Fig. 2. Images of (a) as-deposited solder bumps before reflow and (b) the flip-chip bonded VCSEL array on the quartz substrate. (Color version available online at http://ieeexplore.ieee.org.)

shown in Fig. 1. The VCSEL and photo detector (PD) chips are flip-chip bonded onto the top surface of a transparent substrate, and a polymeric waveguide is formed on the bottom surface of the substrate. In the fabrication of this interconnection plate, the waveguide is formed first and then the VCSEL/PD chips are bonded. If these processes were reversed, the spin-coating of the waveguide materials would be difficult [2]. Thus, to protect
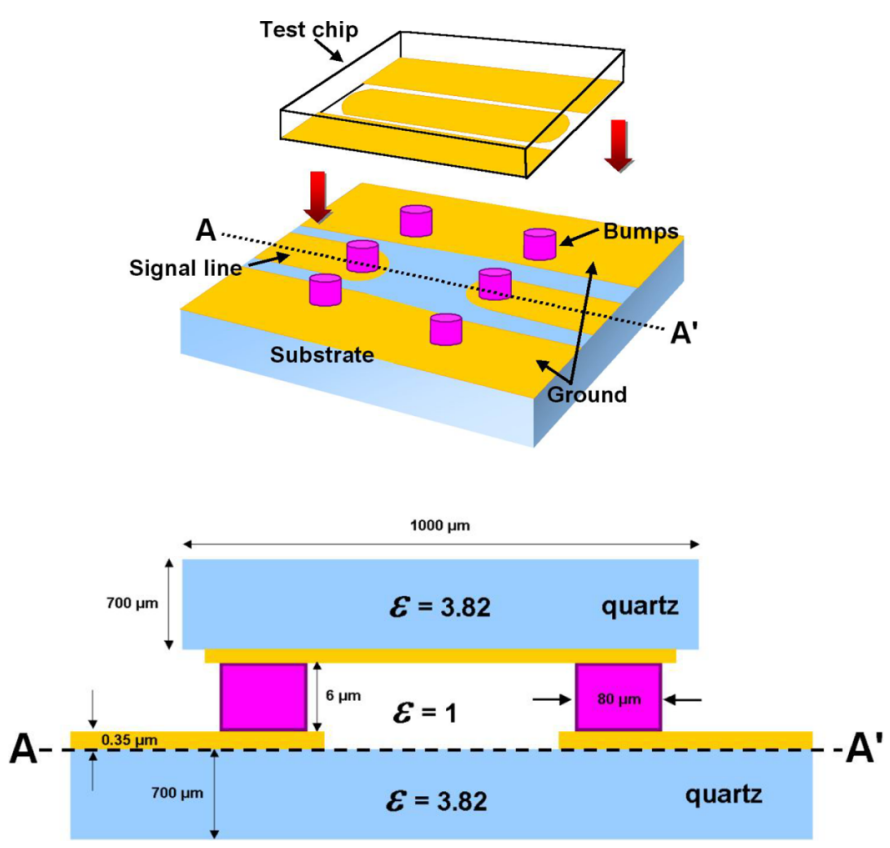

Fig. 3. Flip-chip configuration in $\mathrm{CPW}$ test. $\left(\mathrm{A}-\mathrm{A}^{\prime}\right.$ : cross section of flip-chip assembled CPW package). (Color version available online at http://ieeexplore.ieee.org.)

the polymeric waveguide from thermal damage during flip-chip bonding, a low melting temperature bump material is needed.

In the test sample for the bump metal, metal layers of both Ag-coated indium and uncoated indium were deposited for comparison. The dimensions of the bumps were $6 \mu \mathrm{m}$ in height and $80 \mu \mathrm{m}$ in diameter. The metal lines and bumps are shown in Fig. 2(a). When the VCSEL array chip was flip-chip bonded onto the substrate, the bonding temperatures was $150^{\circ} \mathrm{C}$ and the bonding pressure was 500 gf. The two-channel VCSEL array is $500 \times 470 \mu \mathrm{m}$, with a thickness of $200 \mu \mathrm{m}$. The flip-chip-bonded VCSEL array chip is shown in Fig. 2(b).

\section{B. Fabrication of CPW Flip-Chip Interconnection Samples}

The test sample to measure the microwave characteristics was composed of CPWs on a quartz substrate and a quartz chip, as shown in Fig. 3. The chip was flip-chip bonded onto the substrate using the bumps formed on the substrate, as shown in Fig. 4(a). The flip-chip-bonded CPW sample is shown in Fig. 4(b). The test chip with the dimensions of $1 \times 1 \times 0.7 \mathrm{~mm}$ has $50-\Omega$ CPW through lines. The substrate has the same $50-\Omega$ 


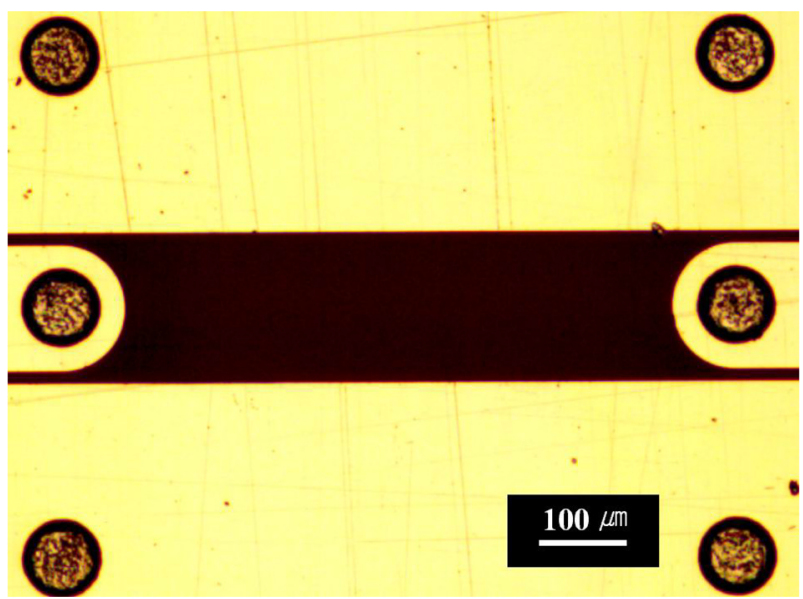

(a)

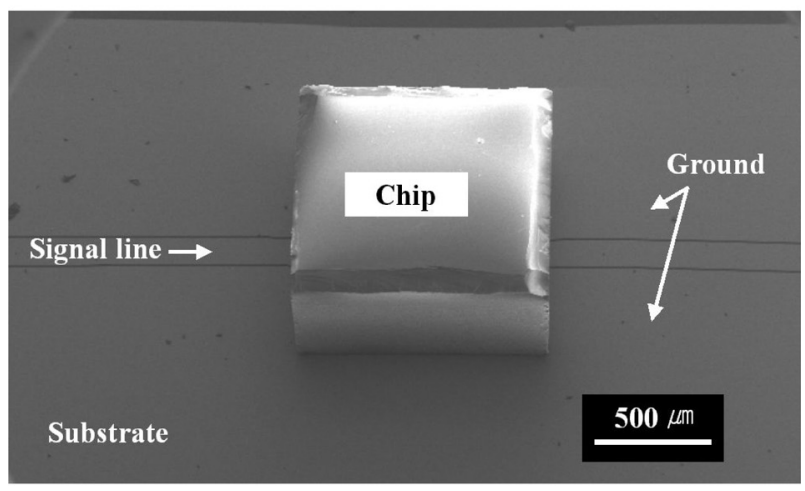

(b)

Fig. 4. Images of (a) as-deposited solder bumps before reflow and (b) the flip-chip bonded CPW structure. (Color version available online at http://ieeexplore.ieee.org.)

TABLE I

Design TABLE FOR THE CPW TEST SyStems

\begin{tabular}{c|c}
\hline Parameter & Value \\
\hline Substrate thickness & $700 \mu \mathrm{m}$ \\
\hline Test chip thickness & $700 \mu \mathrm{m}$ \\
\hline Bump diameter & $80 \mu \mathrm{m}$ \\
\hline Bump shape & Cylindrical \\
\hline Gound-to-ground spacing on substrate & $180 \mu \mathrm{m}$ \\
\hline Line width on substrate & $150 \mu \mathrm{m}$ \\
\hline Gound-to-ground spacing on chip & $180 \mu \mathrm{m}$ \\
\hline Line width on chip & $150 \mu \mathrm{m}$ \\
\hline Dielectric constant & $\mathbf{3 . 8 2}$ \\
\hline Metallization thickness & $0.35 \mu \mathrm{m}$ \\
\hline Total CPW line length (substrate) & $\mathbf{4 ~} \mathbf{~ m m , 1 0 ~} \mathbf{~ m m}$ \\
\hline
\end{tabular}

CPW lines as the test chip. Detailed dimensions and deduced parameters for the 50- $\Omega$ CPW structure are listed in Table I.

\section{Solder Bump Formation and Flip-Chip Process}

The two solder bump materials, for a comparison of the Ag-coated indium and the uncoated indium, were formed in both optical and CPW interconnection samples. In the metal lines, three layers of metal, $\mathrm{Ti}(100 \AA) / \mathrm{Ni}(400 \AA) / \mathrm{Au}(3000 \AA)$

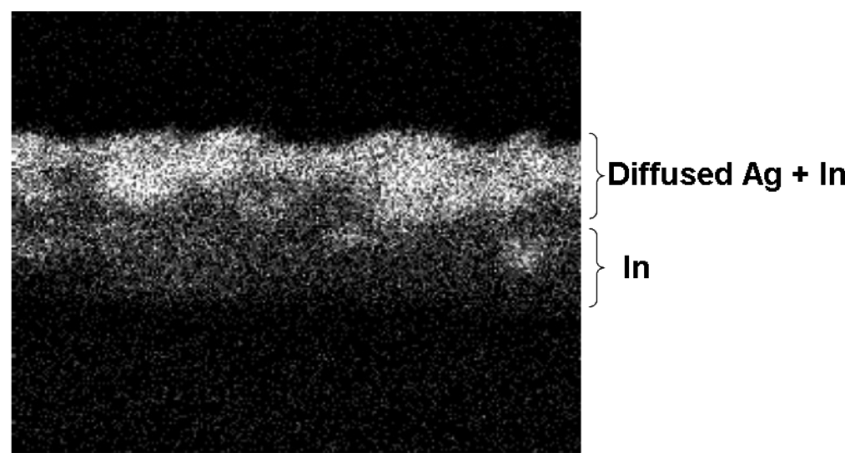

Fig. 5. EDS elemental distribution mapping of diffused $\mathrm{Ag}$ in indium solder.

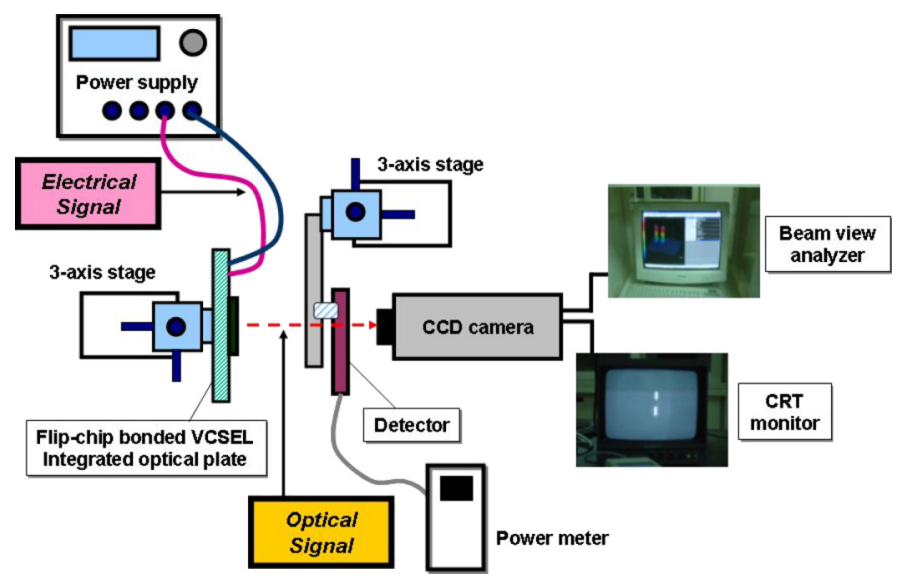

Fig. 6. Experimental setup to measure the light output power of flip-chip-assembled VCSEL array. (Color version available online at http://ieeexplore.ieee.org.)

were deposited via e-beam evaporation. The Ti/Ni/Au layers were used for the under bump metallurgy (UBM). The solder bumps were formed only on the substrate by means of thermal evaporation and lift-off processes. The thickness of the Ag layer coated on the indium was about $2000 \AA$ A . After deposition of solder bump, reflow processing was applied at a temperature above the melting point of indium. The chips were aligned and bonded using a flip-chip bonder (Toray, FC1500 series). During the alignment process, a two-sight camera unit was inserted between the chip and the substrate. After the alignment operation, the bonding head was lowered, and the bonding operation was performed under a specific contact pressure of $500 \mathrm{gf}$ [6].

\section{RESUlTS AND DisCUSSION}

\section{A. Interface of Ag-Coated Indium Solder}

In our previous research [7], it was found that a fracture occurred between indium solder bump and the chip pad during a die shear test. The problem occurred because the bonding temperature was lower than the melting point of indium. The fracture is attributed to the formation of indium oxide, which prevents good adhesion between the indium bump and the VCSEL chip pad. However, a Ag layer coated onto the indium solder bump effectively enhances the adhesion strength between the indium bump and the VCSEL chip pads by decreasing the melting temperature of the indium solder and protecting it from oxidation [5]. In this paper, the alloying state 


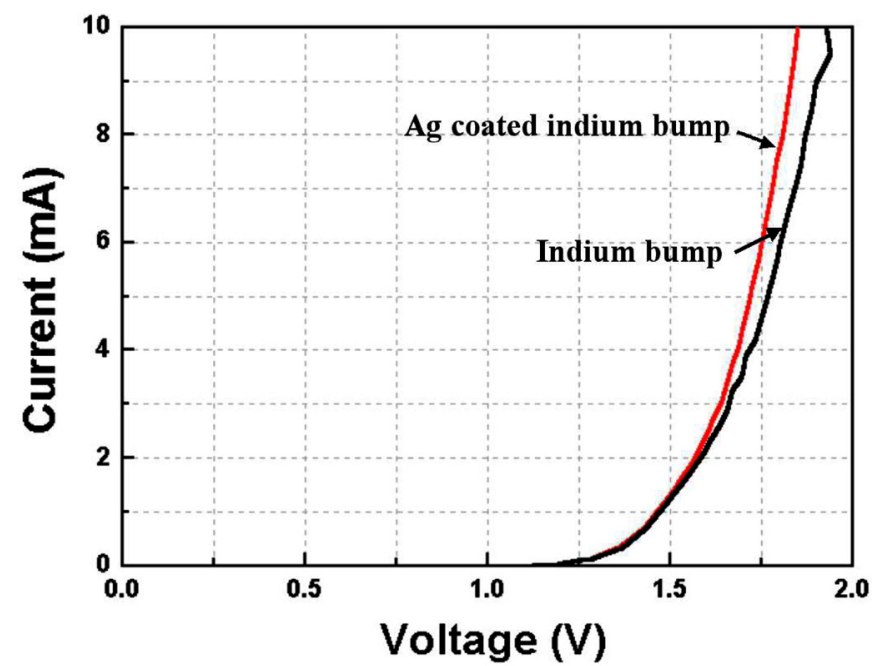

(a)

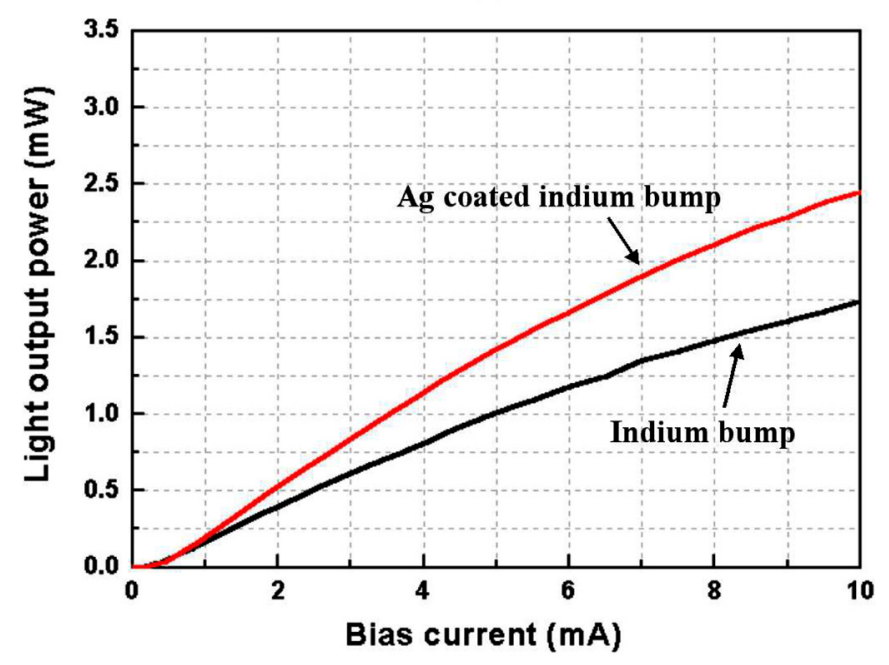

(b)

Fig. 7. Optoelectronic characteristics of flip-chip-bonded VCSEL arrays (a) $I-V$ curves and (b) $L-I$ curves. (Color version available online at http://ieeexplore.ieee.org.)

of the $\mathrm{Ag} / \mathrm{In}$ interface is analyzed. Fig. 5 shows the energy dispersive spectroscopy (EDS) elemental distribution mapping of diffused $\mathrm{Ag}$ in an indium solder. It can be inferred that the $\mathrm{Ag}$ atoms are deeply diffused into the indium solder, despite the small amount of $\mathrm{Ag}$ involved. In general, the Ag diffuses rapidly into indium via an interstitial mechanism, and indium diffuses into silver through grain boundaries with a diffusion coefficient $D_{0}$ of $2.4 \times 10^{-12} \mathrm{~m}^{2} \mathrm{~s}^{-1}$ and an activation energy of $0.42 \mathrm{eV}$ [8]. Therefore, immediately after deposition, the $\mathrm{Ag}$ layer interacts with the indium to form a $\mathrm{AgIn}_{2}$ compound layer due to the high interdiffusion coefficient [9]. Formation of a $\mathrm{AgIn}_{2}$ compound was identified from scanning electron microscopy (SEM) and EDS analyses.

\section{B. Optoelectronic Characteristics of Flip-Chip-Bonded VCSEL Array}

Fig. 6 shows the experimental setup used to measure the light output power of the flip-chip assembled VCSEL array. The characteristics of the VCSEL arrays that were flip-chip bonded using

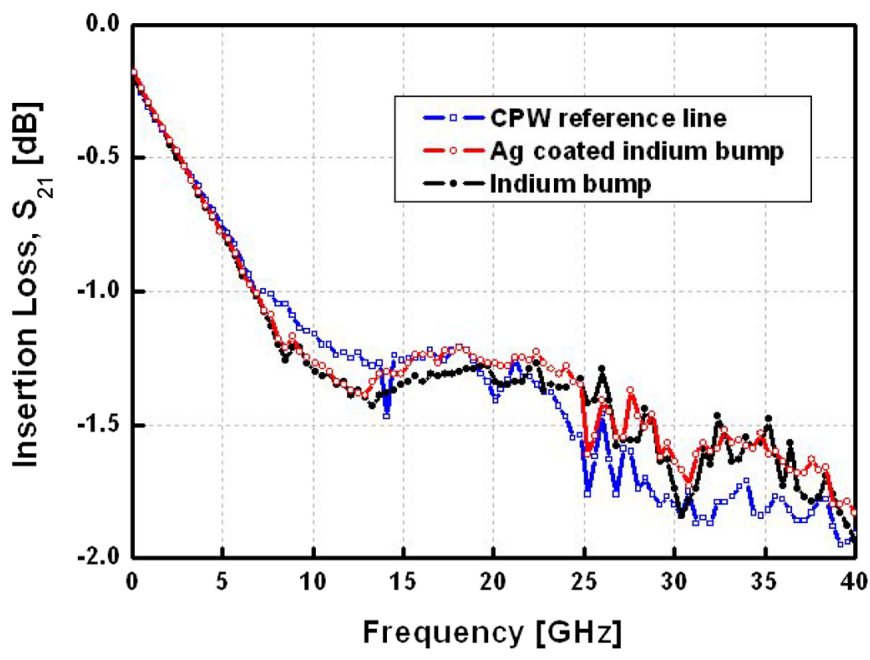

(a)

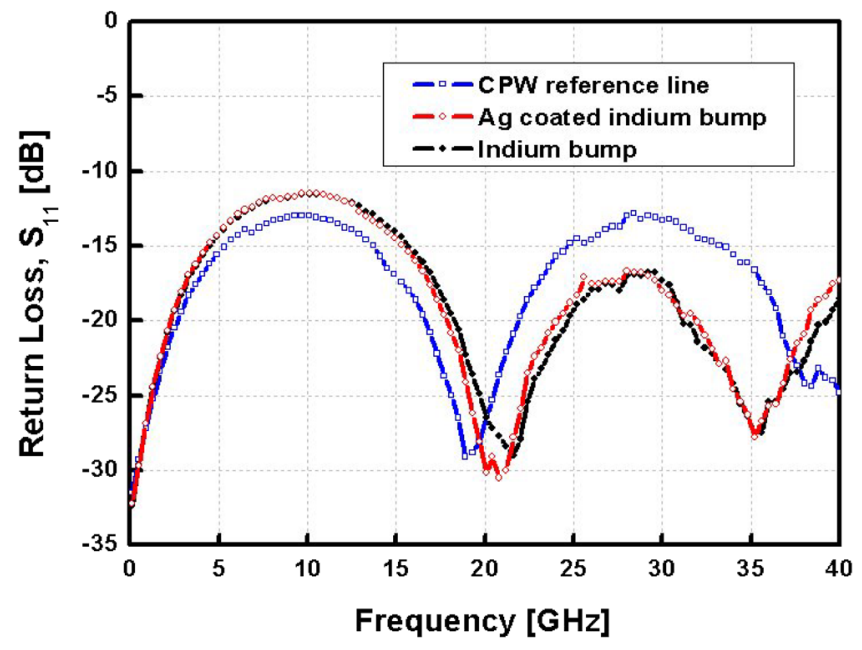

(b)

Fig. 8. Measured (a) insertion loss $\left(\mathrm{S}_{21}\right)$ and (b) return loss $\left(\mathrm{S}_{11}\right)$ for indium bump and Ag-coated indium bump (total CPW line length: $4 \mathrm{~mm}$ ). (Color version available online at http://ieeexplore.ieee.org.)

either a Ag-coated indium bump or an uncoated indium bump are compared by means of $I-V$ and $L-I$ inspections. In this paper, two-channel VCSEL arrays operating at 850-nm wavelength range with rates of $2.5 \mathrm{~Gb} / \mathrm{s}$ were used. Fig. 7(a) shows the $I-V$ curves of the flip-chip-bonded VCSEL. It inferred that the resistance of a Ag-coated indium bump is slightly lower than that of the uncoated indium bump. The $L-I$ characteristics of the VCSELs were compared in Fig. 7(b). The light output power of the Ag-coated indium bump is higher than that of the indium bump. In addition, the Ag coating on the indium bumps decreases the threshold current of the VCSEL from 50 to $43 \mathrm{~mA}$. Therefore, the $I-V$ and $L-I$ curves indicate that the Ag-coated indium bumps are superior to the uncoated indium solder bumps with regard to optical and electrical performances.

\section{Characterization of a Flip-Chip Interconnect at Frequencies Up to $40 \mathrm{GHz}$}

The RF and microwave characteristics of the flip-chipbonded CPW samples were analyzed for the entire frequency 


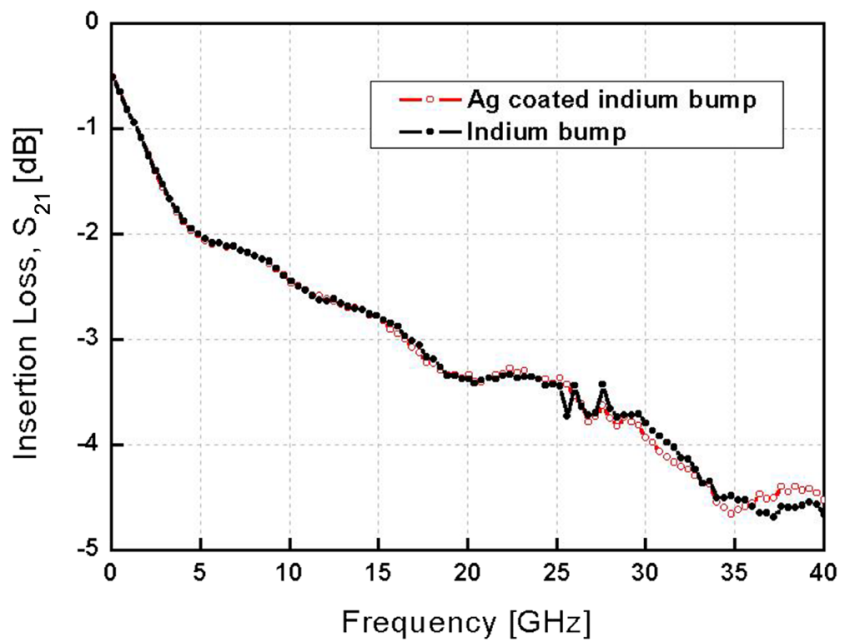

(a)

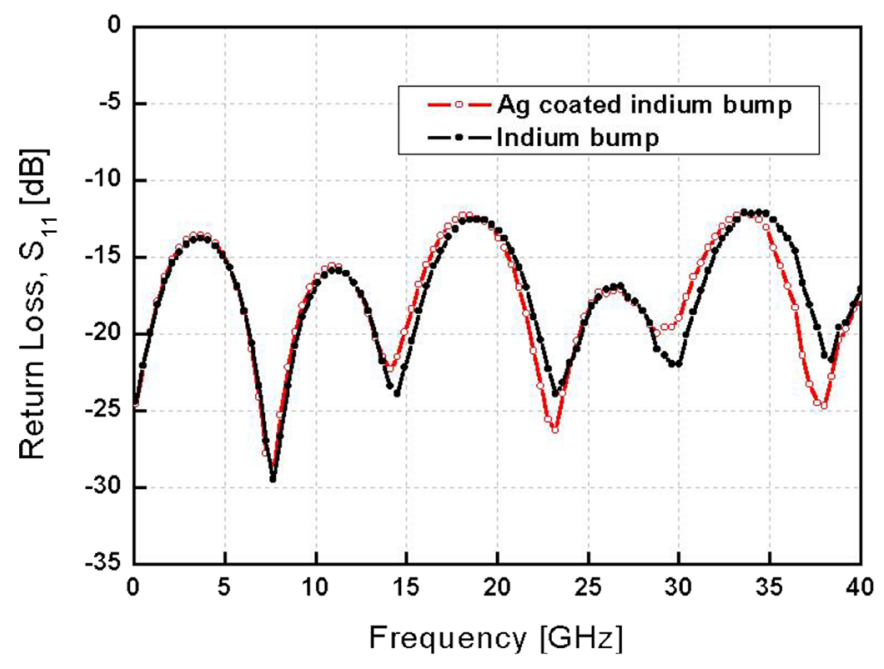

(b)

Fig. 9. Measured (a) insertion loss $\left(S_{21}\right)$ and (b) return loss $\left(S_{11}\right)$ for indium bump and Ag-coated indium bump (total CPW line length: $10 \mathrm{~mm}$ ). (Color version available online at http://ieeexplore.ieee.org.)

range from 0.1 to $40 \mathrm{GHz}$. It has been reported that the RF performance of the flip-chip bonded structure is influenced by different bump materials [10], [11]. In this paper, the effect of the Ag coating on the indium bumps on the RF performances was investigated.

The measurement of the scattering parameters was performed using an Agilent 8722ES network analyzer with an on-wafer probe station for the frequency range up to $40 \mathrm{GHz}$. Figs. 8 and 9 show the measured reflection and insertion losses, in terms of frequency, corresponding to total CPW lengths of 4 and $10 \mathrm{~mm}$, respectively. In both cases, when the total CPW line length is $10 \mathrm{~mm}$, the insertion loss is about $3.3 \mathrm{~dB} / \mathrm{cm}$ at $20 \mathrm{GHz}$ and the return loss is less than $13 \mathrm{~dB}$, as shown in Fig. 9. The microwave transmission effect of the Ag coating is negligible by comparison with uncoated indium bumps. The measured results for the CPW only are an insertion loss of about $3.3 \mathrm{~dB} / \mathrm{cm}$ at $20 \mathrm{GHz}$ and a reflection loss of less than $14 \mathrm{~dB}$. Thus, both Ag-coated and uncoated indium solder bumps can be used in flip-chip interconnects, while preserving the original characteristics of CPW signal lines.

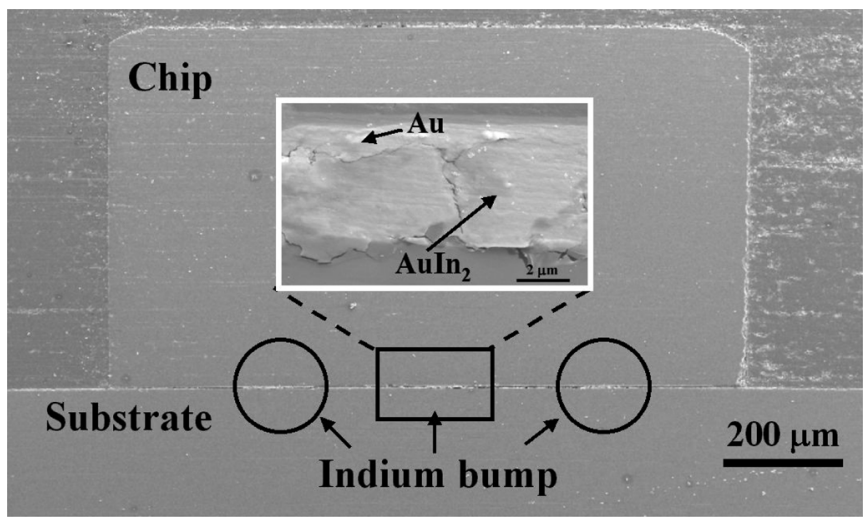

(a)

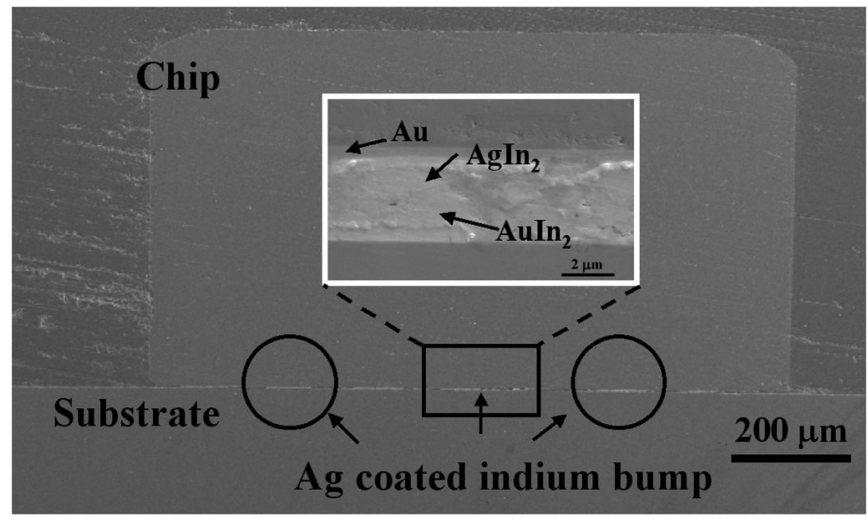

(b)

Fig. 10. Joint cross-sectional SEM images of CPW package using (a) indium and (b) Ag-coated indium bumps.

\section{Cross Sections of Flip-Chip Assembled CPW Samples}

SEM and EDS analyses were performed on the joint cross sections of the CPW packages. Fig. 10(a) and (b), shows the joint cross-sectional SEM images of the CPW package using indium and Ag-coated indium bumps, respectively. The SEM and EDS results indicate that the joint [Fig. 10(a)] consists of an indium-gold alloy with embedded intermetallic grains of $\mathrm{AuIn}_{2}$. In addition, SEM and EDS results indicate that the joint [Fig. 10(b)] consists of an In-Au-Ag mixture alloy with embedded intermetallic grains [5]. The joint microstructure of the Ag-coated indium solder is similar to that of indium solder. The height of the solder bump was changed from 6.0 to $3.6 \mu \mathrm{m}$ after the flip-chip bonding process.

\section{CONCLUSION}

This paper presents low-temperature flip-chip bonding for both optical interconnect and microwave applications, comparing the use of a Ag-coated indium bump and an uncoated indium bump. Coating a thin $\mathrm{Ag}$ layer onto the indium surface enhanced the optoelectronic characteristics of an indium bump. However, the effects of the Ag coating on microwave characteristics are negligible. Both the Ag-coated and uncoated indium solder bumps maintain the original characteristics of CPW signal lines almost without additional losses from the flip-chip-bonded bumps. 


\section{REFERENCES}

[1] A. Koschel and S. Cyrusian, "Comprehensive characterization of flip chip contacting methods for microwave and optoelectronic applications," in Proc. SBMO/IEEE MTT-S IMOC, 1995, pp. 340-345.

[2] H. S. Cho, K.-M. Chu, S. K. Kang, B. S. Rho, W. H. Kim, J.-S. Kim, J.-J. Kim, and H.-H. Park, "Compact packaging of optical and electronic components for on board optical interconnects," IEEE Trans. Adv. Packag., vol. 28, no. 1, pp. 114-120, Feb. 2005.

[3] S. Choe, W. W. So, and C. C. Lee, "Low temperature fluxless bonding technique using In-Sn composite," Proc. Electron. Compon. Technol. Conf., pp. 114-118, 2000.

[4] Y. C. Chen, W. W. So, and C. C. Lee, "A fluxless bonding technology using indium-silver multiplayer composites," IEEE Trans. Compon., Packag., Manuf. Technol. A, vol. CPMT-20, no. 1, pp. 46-51, Mar. 1977.

[5] K.-M. Chu, J.-S. Lee, H. S. Cho, H. H. Park, and D. Y. Jeon, "A fluxless flip chip bonding for VCSEL arrays using silver-coated indium solder bumps," IEEE Trans. Electron. Packag. Manuf., vol. 27, no. 4, pp. 246-253, Oct. 2004

[6] T. Suga, T. Itoh, Z. Xu, M. Tomita, and A. Yamauchi, "Surface-activated bonding for new flip chip and bumpless interconnect systems," in Proc. Electron. Compon. Technol. Conf., 2002, pp. 105-111.

[7] K.-M. Chu, J.-S. Lee, H. S. Cho, B. S. Rho, H.-H. Park, and D. Y. Jeon, "Characteristics of indium bump for flip-chip bonding used in polymeric-waveguide-integrated optical interconnection systems," Jpn. J. Appl. Phys., vol. 43, no. 8B, pp. 5922-5927, 2004.

[8] V. Simic and Z. Marinkovic, "Room temperature interactions in $\mathrm{Ag}$ metals thin film couples," Thin Solid Films, vol. 61, pp. 149-160, 1979.

[9] R. Roy and S. K. Sen, "The kinetics of formation of intermetallics in Ag/In thin film couples," Thin Solid Films, vol. 197, pp. 303-318, 1992.

[10] J. Heyen, J. Schroeder, and A. F. Jacob, "Low-cost flip-chip alternatives for millimeter wave applications," in IEEE MTT-S Dig., 2002, pp. 2205-2208.

[11] J. M. Vahter, J. J. Heikkinen, J. J. Tanskanen, A. K. Seppala, and E. O. Ristolainen, "Rf-performance of a low-cost flip chip assembly," in Proc. IPACK, 2001. 15609.

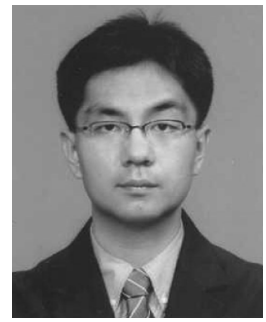

Kun-Mo Chu (S'06) received the B.S. degree in materials science and engineering from Korea University, Seoul, in 2001 and the M.S. degree in materials science and engineering from the Korea Advanced Institute of Science and Technology (KAIST), Daejeon, in 2003, where he is currently pursuing the Ph.D. degree.

$\mathrm{He}$ is also a Research Assistant with the Optical Interconnection and Switching Laboratory, Information and Communications University, Daejeon. His research interests include optical interconnection architecture and flip-chip technology for electronic and photonic devices.

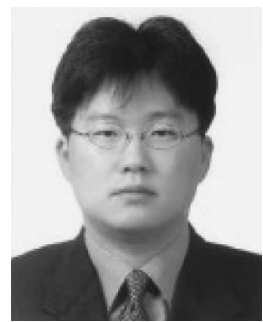

Jung-Hwan Choi received the B.S. degree in computer engineering and electrical engineering from Handong University, Pohang, Korea, in 2002 and the M.S. degree in electrcal engineering from the Information and Communications University, Daejeon, Korea, in 2005, where he is currently pursuing the $\mathrm{Ph} . \mathrm{D}$. degree.

His research interests include millimeter/microwave measurement systems.

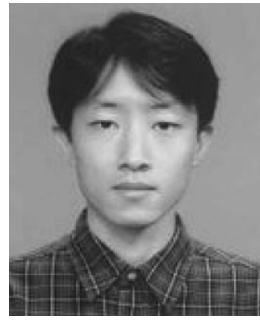

Jung-Sub Lee received the B.S. degree in materials science and engineering from the Korea Advanced Institute of Science and Technology (KAIST), Daejeon, in 2002, where he is currently pursuing the Ph.D. degree.

He is also a Research Assistant with the Optical Interconnection and Switching Laboratory, Information and Communications University, Daejeon. His research interests include flip-chip technology and solder bumping processes in SIP systems.

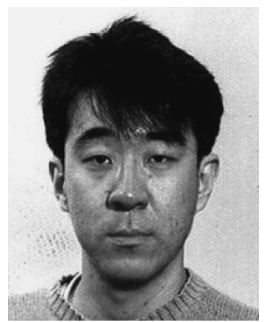

Han Seo Cho received the Ph.D. degree in materials science and engineering from the Korea Advanced Institute of Science and Technology (KAIST), Daejeon, in 1999.

He worked as a Postdoctoral Researcher with The Johns Hopkins University, Baltimore, MD, until 2001. He is currently a Research Professor in the Information and Communications University (ICU), Daejeon, , and his research interests include optical material characterization, design and demonstration of chip-to-chip optical interconnection using electrooptical printed circuit boards (EOCBs). He is also with the Samsung Electro-Mechanics Corporation, Ltd., Chungnam, Korea.

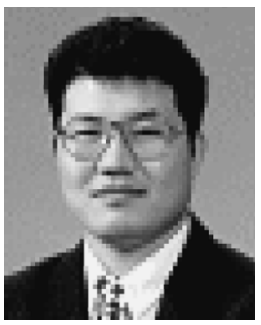

Seong-Ook Park received the Ph.D. degree in electrical engineering from Arizona State University (ASU), Tempe, in 1997.

From March 1989 to August 1993, he was a Research Engineer with Korea Telecom, Daejeon, working with microwave systems and networks. He later joined the Telecommunication Research Center, ASU, until his departure in September 1997. Since October 1997, he has been with the Information and Communications University, Daejeon, as an Associate Professor. His research interests include $4 \mathrm{G}$ handset antennas, millimeter/microwave systems, and analytical and numerical techniques in the area of electromagnetics.

Dr. Park is a member of the Phi Kappa Phi.

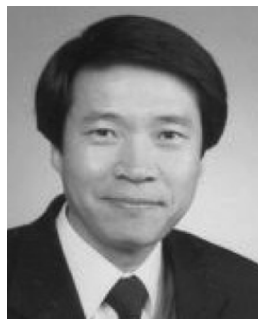

Hyo-Hoon Park (M'94) received the Ph.D. degree in material science and engineering from the Korea Advanced Institute of Science and Technology (KAIST), Daejeon, in 1985.

From 1985 to 1986, he worked in the area of compound semiconductor devices at Stanford University, Stanford, CA, as a Postdoctoral Scholar. From 1986 to 1997, he worked at the Electronics and Telecommunications Research Institute in area of high-speed electronic devices and vertical-cavity surface-emitting lasers. Since 1998, he has been a Professor in the Optical Communication Group, Optical Interconnection and Switching Laboratory, Information and Communications University (ICU), Daejeon. Since joining ICU, he has been studying optical interconnection for chip-to-chip and board-to-board data links, optical transceiver modules, and optical switching components for optical communication systems and networks.

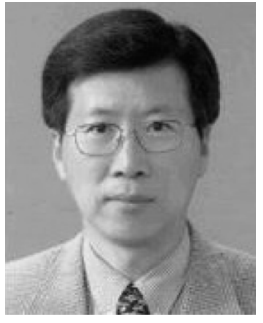

Duk Young Jeon received the B.S. degree from Seoul National University, Seoul, Korea, in 1976, the M.S. degree in applied physics from the Korea Advanced Institute of Science and Technology (KAIST), Daejeon, in 1979, and the Ph.D. degree in physics from Lehigh University, Bethlehem, PA, in 1988.

In 1988, he joined AT\&T Bell Laboratories, Allentown, PA, as a Member of Technical Staff, where he was involved in the development of advanced lithography for submicron CMOS technology. Later, he was involved in researching gate technology for $0.1-\mu \mathrm{m}$ CMOS, and plasma processing and device study for the high-speed BiCMOS technology at AT\&T, Holmdel, NJ, and backend integration for deep submicrometer modular BiCMOS technology at AT\&T, Murray Hill, NJ. Since 1994, he has been with KAIST, as a Professor of Materials Science and Engineering. His research interests are in display materials and devices, such as phosphors, carbon nanotubes, optical packaging, and optical MEMs devices. 